\title{
Rehabilitation Assessment Based on the Complexity and Fluctuation of EMG Signal
}

\author{
Qingsong $\mathrm{Ai}^{1,2, \mathrm{a}}$, Ling Chen ${ }^{1,2, \mathrm{~b}}$, Wei Meng ${ }^{1,2, \mathrm{c}}$ \\ ${ }^{1}$ School of Information Engineering, Wuhan University of Technology, 430070, Wuhan, China \\ ${ }^{2}$ Key Lab. of Fiber Optic Sensing Technology and Information Processing, 430070, Wuhan, China \\ aqingsongai@whut.edu.cn, bchenlingwhut@163.com, cweimeng@whut.edu.cn
}

Keywords: Rehabilitation assessment, EMG, complexity, embedding dimension, fluctuation

\begin{abstract}
The assessment after rehabilitation is an important part of rehabilitation medicine, and of great significance for the basic research and clinical application for rehabilitation medicine. Physician can make objective analysis about patients' present conditions, and then appropriate training program can be developed for patients to recover their body function more effectively. Three kinds of EMG signal, that is, the healthy, patient with myopathy, and patient with neuropathy, are involved. The complexity of the three types of signal is compared and the related variables (embedding dimension and time delay of phase space reconstruction) are determined by singular-value decomposition and mutual information method. Moreover, a novel effective approach improved from Hilbert-Huang Transform (HHT) is proposed here for the further comparision among the three EMG signals. The results show both methods can distinguish the three kinds of EMG signal clearly.
\end{abstract}

\section{Introduction}

Rehabilitation therapy brings significant benefits to individuals who recover from a wide range of surgeries, illnesses and injuries. Rehabilitation evaluation and assessment can greatly contribute to assess the control strategy, and make it possible to produce more novel and cost-effective solutions to improve the outcome of rehabilitation process. Rehabilitation evaluation based on clinical scale assessment, physical quantitative assessment, and biological signals-based assessment respectively, are the most widely utilized aspects. However, the rehabilitation results always depend largely on therapist's experience and it is hard to detect the subtle differences before and after the rehabilitation by using clinical scale assessment and physical quantitative assessment methods which depend on several subjective factors, e.g. the experience of physicians. In contrast, EMG signal is capable of reflecting the natural characteristic when patient moves or has the intention to move, and it makes EMG signal more necessary as a tool to assess the body function after rehabilitation.

The work in this paper is about to distinguish three kinds of EMG signal from healthy, patient with myopathy and patient with neuropathy. In section II, related works about rehabilitation assessment in recent years are introduced. Section III is about the complexity of three kinds of EMG signal, and a novel method (i.e. fluctuation) based on Hilbert-Huang transform (HHT) is presented in the following section. Finally, conclusion is drawn in section V.

\section{Related Works}

There has been a wide range of evaluation criteria for rehabilitation, especially with the introduction of robotic technologies in medical filed recently, where modern medicine is based on objective evaluation and quantitative comparative analysis of the impact of different therapeutic approaches [1]. These methods for quality assessment of rehabilitation can be divided into three categories according to the data resources recorded, including clinical scale assessment, physical quantitative assessment, and biological signals-based assessment. 
The clinical scale assessment is mainly performed by using international standard of rehabilitation rating scales, such as the simplified Fu-Meyer Assessment (FMA), Composite Spasticity Scale (CSS), Functional Ambulation Category (FAC), Functional Independence Measure (FIM), and Postural Assessment Scale (PASS) [2, 3]. The efficacy of the training method is assessed through real-time experiments, and the corresponding scores can be obtained based on the actual execution outcomes following the desired motions with variable limb speed profiles [4]. However, the objectivity and reliability of this methodology is widely questioned, due to the therapist's experience and patient's mental state which will impose extremely adverse influence on the rehabilitation assessment results.

The physical quantitative assessment mainly depends on the physical parameters recorded during the rehabilitation process, such as the angle of joint, force or torque of arm, speed, tremor, etc. Casadio et al. defines four performance indicators or outcome measures [5] to analyze and assess the hemiplegias' rehabilitation outcomes. Literature [6] completes the rehabilitation evaluation of the effect of robot-assisted gait training through several physical parameters including gait speed, rhythm, and walking duration, etc. Furthermore, the combination of surface EMG and robotic control can be found in [7]. These approaches based on physical state, especially the kinematics and dynamics of the patient's limb, are able to provide a more precise assessment than the scale method. On the other hand, it is difficult to record all minor changes of these physical parameters during the rehabilitation process, which tends to cause inaccurate reflection of the real disabled situation.

EMG signal contains rich information, from which a user's intention and the fatigue-like states in the form of muscular contraction can be detected by using surface electrodes [8]. And it is revealed that the muscle fiber contraction can be reflected by the rise of root mean square (RMS) of surface EMG [9]. In [10], the electrodes are placed on the tibialis anterior and gastrocnemius to record the maximum isometric contraction in the process of ankle dorsiflexion and plantar flexion. [11] utilizes the median frequency (MF) and mean power frequency (MPF) of EMG to detect the muscle fatigue, and the results show that MF will be significantly reduced if the fatigue occurs, and the MF value after training will be marginally larger than before. Because of its advantages in overcoming the poor reliability and low sensitivity of traditional assessment approaches, the biological signal-based method has become one of the most important parts in modern rehabilitation medicine domain. In addition, this method is able to simplify the evaluation process, shorten the assessment duration, and has the potential to improve the rehabilitation efficiency significantly.

\section{EMG Signal Collection}

Electromyogram (EMG) is very common to be utilized as an effective tool to evaluate the muscles and the nerves that control them. Here, we use EMG signal to distinguish the disparity of healthy individuals and the diseased ones. More specifically, the EMG signal involved in this research is collected from three people, as show in Table 1.

Table 1. Detail information about where the EMG signal comes from

\begin{tabular}{|c|c|c|c|}
\hline Person & Age(year old) & Gender & Description \\
\hline 1 & 44 & male & Without history of neuromuscular disease \\
\hline 2 & 57 & male & $\begin{array}{c}\text { With myopathy due to longstanding history of } \\
\text { polymyositis, treated effectively with steroids } \\
\text { and low-dose methotrexate }\end{array}$ \\
\hline 3 & 62 & male & $\begin{array}{r}\text { With chronic low back pain and neuropathy } \\
\text { due to a right L5 radiculopathy }\end{array}$ \\
\hline
\end{tabular}

The whole datasets are collected at $50 \mathrm{KHz}$ before downsampled to $4 \mathrm{KHz}$. A $20 \mathrm{~Hz}$ high-pass filter and a $5 \mathrm{KHz}$ low-pass filter are used during the recording process. Fig. 1 shows the collected EMG signal in a certain period of time. 

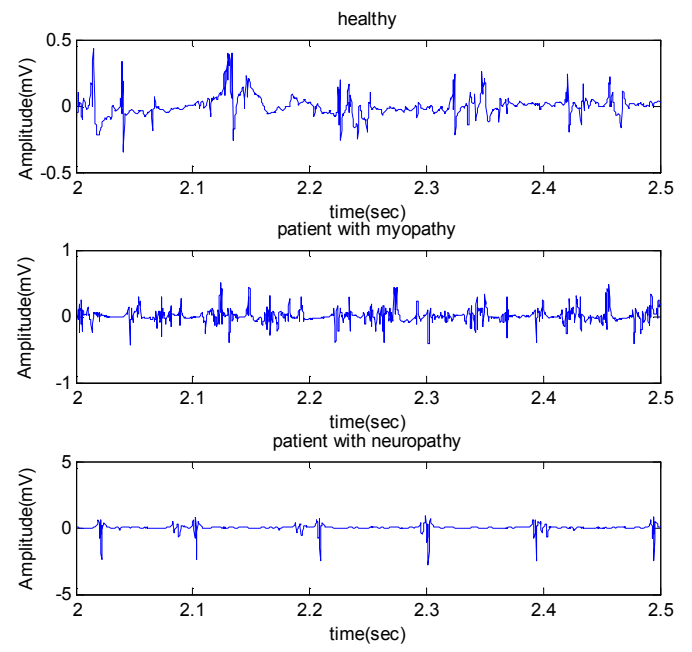

Fig. 1. Waveform of three different types of EMG signal

In order to analyze the signal in more detail, signal is segmented by every 0.5 second. And the same number of datasets (24) for the three kinds of EMG signal are analyzed and there are 2000 data points for each dataset.

\section{Complexity of EMG Signal}

The singular-value decomposition method for determining the embedded dimension $m$ are commonly utilized by choosing an appropriate $M$ to define an $M^{*} M$ autocorrelation matrix. Fig.2 demonstrates the result of three kinds of EMG signal and the pure white noise as well (for contrast). For pure white noise, the singular values remain stable along with the change of $M$, while for all kinds of EMG signal, their singular values start to stabilize after $M$ reaches 15 . Therefore 15 is proposed for the embedding dimension for all the three types of EMG signal. Besides, the choice of time delay $\tau$ is confirmed by using mutual information.

$$
I(\tau)=\sum_{k=1}^{N} P[y(k), y(k+\tau)] \log \left\{\frac{P[y(k), y(k+\tau)]}{P[y(k)] P[y(k+\tau)]}\right\} \geq 0 .
$$

The probabilities of two time series corresponding two measurement variables are $P[x(k)]$ and $P[x(k+\tau)]$ respectively. And $P[x(k), x(k+\tau)]$ is the probability of the result when measuring $x(k)$ and $x(k+\tau)$ together. This method is applied to confirm time delay and the result is exhibited in Fig. 3 which shows that the value of time delay is promising when designed as 10 .
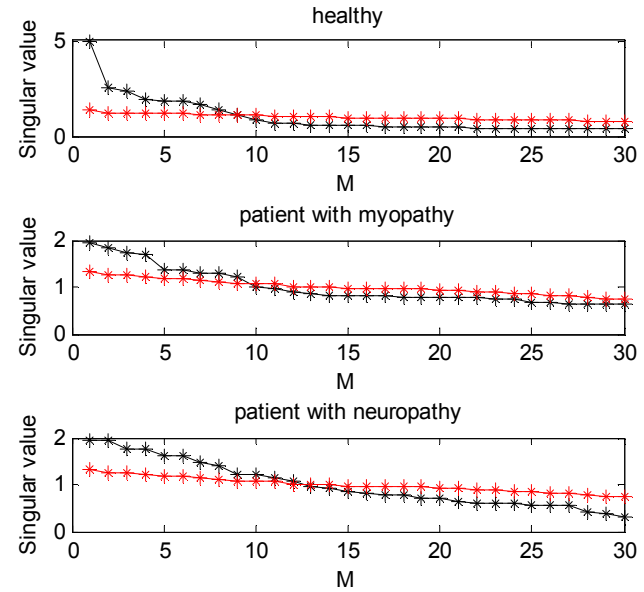
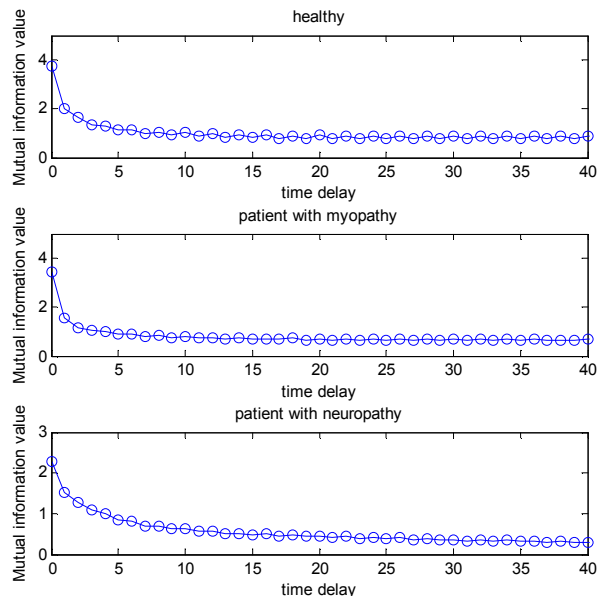

Fig. 2. Singular-value decomposition method for $\mathrm{m}$ Fig. 3. Mutual information method for $\tau$ 
The complexity of signal describes the randomness and the disorder regarding to the internal structure of signal. By using complexity, it is of efficacy to reflect the features of the signal itself. Based on the information entropy, R. Lopez-Ruiz et al. have defined statistical complexity shown as

$$
\text { Complexity }(x)=H(x) * D(x) . \quad D(x)=\sum P(x) \log _{2}[P(x) / \widehat{P}(x)] .
$$

where $H(x)$ is Shannon entropy while $D(x)$ is an improved intensive quantity.

With regards to variable $\mathrm{P}(\mathrm{x})$ and $\hat{P}(x)$ (probability of a particular event and probability of uniform distribution, respectively), a new and effective method is proposed in this paper to determine them.

Firstly we reconstruct the phase space of the signal based on nonlinear dynamics theory, after normalizing the data to the range of $[0,1]$. The mean value and the standard deviation of each phase space after reconstruction are calculated. Afterwards, the mean value and the standard deviation series of signal are divided into equal segment, separately. And the length of the segment is stated as

$$
\text { Length }=(\max (\text { Values })-\min (\text { Values })) / \text { totalsegments } .
$$

where totalsegments is designed as 10 , while Values represents the mean value or the standard deviation series. The principle for choosing the sections is shown in Fig. 4, so that we can get the different probabilities for different sections. The number of data points satisfied the section $i$ are described as Number $(i)$. And the total number of these data points is defined as Number(total). Eventually, the corresponding probability for each section is obtained by the following formula

$$
\begin{aligned}
& \text { Number(i)=length[LM(i-1)<data }<\operatorname{LM}(\mathrm{i}) \text { and } \operatorname{LS}(\mathrm{i}-1)<\text { data }<\mathrm{LS}(\mathrm{i})] . \\
& P(i)=\operatorname{Number}(i) / \operatorname{sum}(\text { Number }(i)), i=1,2, \ldots \ldots, 10 .
\end{aligned}
$$

Fig. 5 demonstrats that the signal's complexity of healthy people is much less than that of patient with neuropathy, and marginally less than that of patient with myopathy as well. In comparison, the complexity of patient with neuropathy is much higher than that of the other two types of EMG signal.
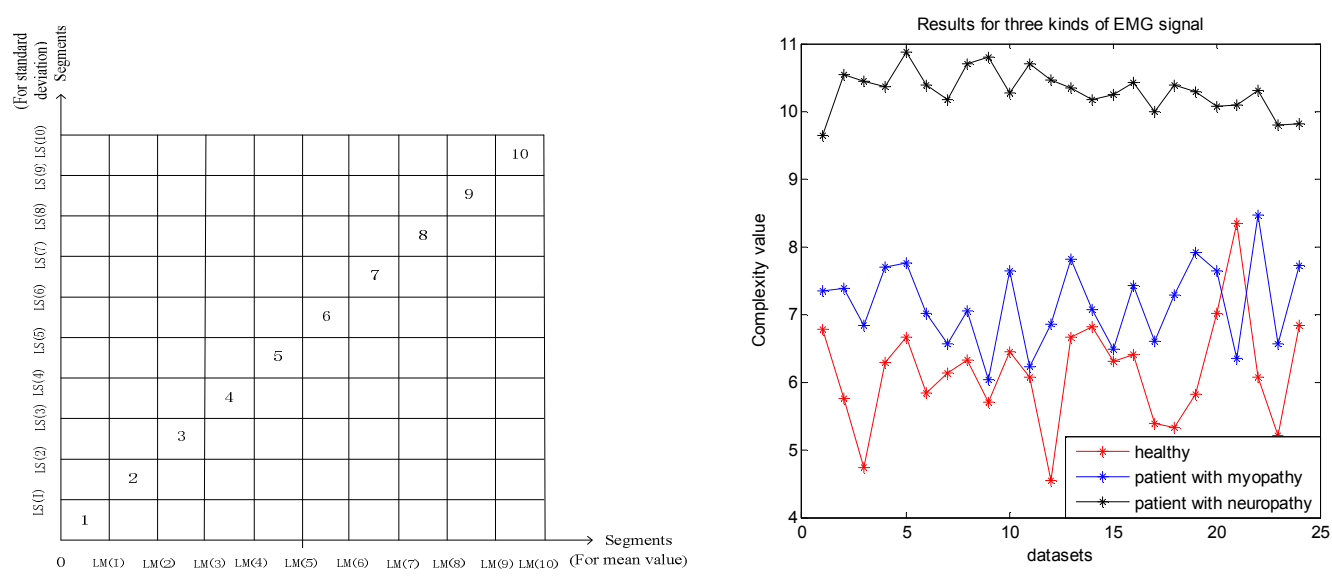

Fig. 4. The principle for choosing sections

Fig. 5. Complexity of three kinds of EMG signal

\section{Fluctuation of EMG}

The volatility of signal can reflect the stability of signal, so that it is possible for us to characterize different categories of signals according to this property. Traditional data-analysis methods are all based on linear and stationary assumptions. Only in recent years have new methods been introduced to analyze nonlinear data [16]. For example, a recently developed method, the HHT, seems to be able to meet some challenges for processing such data.

HHT is designed to obtain the overall trend of signal by removing redundant information in every layer according to the method of envelope operating. But it is inefficient to reflect the fluctuation of signal. Referring for HHT, we can get a new data calculated by using the difference of two adjacent 
raw data point. In order to meet the definition of local volatility, the same processing measure needs to be repeated certain amount of times. Supposing $u(t)$ and $u(t-1)$ are the value of two adjacent data point of raw signal $x(t)$. The process of the whole calculation is provided as

$$
\begin{gathered}
h_{1}(t)=\left[u_{1}(t)-u_{1}(t-1)\right], \quad h_{2}(t)=\left[h_{1}(t)-h_{1}(t-1)\right], \ldots, h_{k}(t)=\left[h_{k-1}(t)-h_{k-1}(t-1)\right] . \\
F(t)=h_{k}(t), r(t)=x(t)-F(t)
\end{gathered}
$$

Until $h_{k}(t)$ satisfies the feature of fluctuation. After that, the final evaluation signal $F(t)$ and the rest of the signal $r(t)$ are obtained.

The new data set for each kinds of EMG signal after 200 times processing is illustrated in Fig. 6. Afterwards, for each dataset, both the maximum value and the average value of the corresponding new dataset are gained before the next processing step(newdata is the data value of signal $F(t)$ ).

$$
\begin{gathered}
\text { if } \operatorname{abs}(\text { newdata }(i)>\operatorname{mean}(\text { newdata })) \text { and } \operatorname{abs}(\text { newdata }(i)>=\text { factor } * \max (\text { newdata })) \\
\operatorname{Newdata}(i)=\operatorname{newdata}(i) \\
\text { else } \operatorname{Newdata}(i)=0 .
\end{gathered}
$$

where factor is set as 0.2.And the number of the non-zero point value of Newdata can represent the fluctuation of EMG signal. It can be seen clearly from the Fig. 7 that EMG signal collected from patient with myopathy has higher fluctuation value compared with those from heathy and patient with neuropathy whereas the fluctuation of patient with neuropathy has the least value.
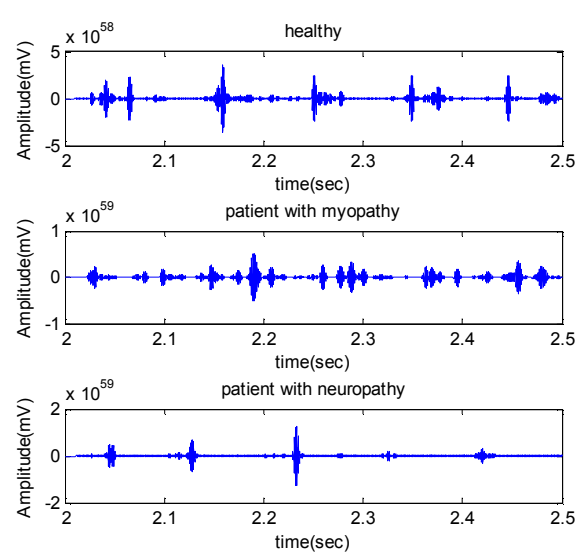

Fig. 6. New data after 200 times processing

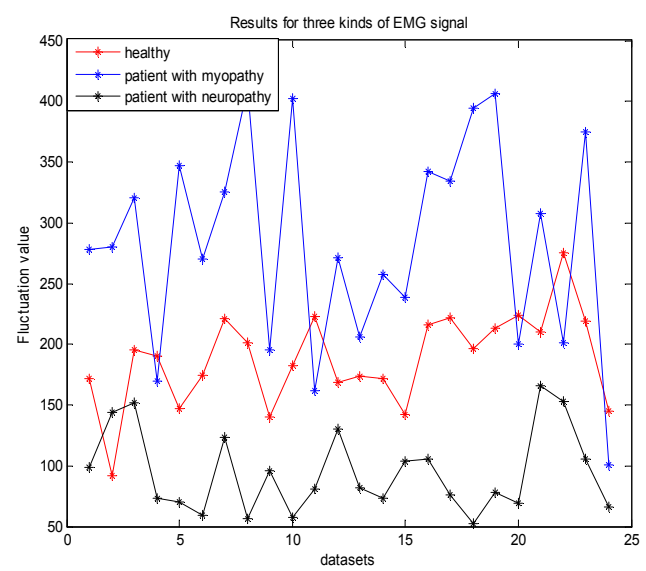

Fig. 7. Fluctuation of three kinds of EMG signal

\section{Discussion and Conclusion}

The assessment after rehabilitation is of vital importance for the clinical application and an indispensable part of rehabilitation medicine. Three kinds of EMG signal (the healthy, patient with myopathy and patient with neuropathy) are studied. Table 2 and table 3 are the corresponding compared information about the complexity and fluctuation of these three kinds of EMG signal.

Table 2 Compared information about complexity

\begin{tabular}{|c|c|c|c|}
\hline Complexity value & Maximum & Minimum & Average \\
\hline healthy & 8.35 & 4.54 & 6.14 \\
\hline patient with myopathy & 8.46 & 6.03 & 7.15 \\
\hline patient with neuropathy & 10.86 & 9.64 & 10.30 \\
\hline
\end{tabular}

Table 3 Compared information about fluctuation

\begin{tabular}{|c|c|c|c|}
\hline Fluctuation value & Maximum & Minimum & Average \\
\hline healthy & 275 & 92 & 188.17 \\
\hline patient with myopathy & 411 & 101 & 283.08 \\
\hline patient with neuropathy & 166 & 52 & 94.63 \\
\hline
\end{tabular}


From all the results, we can conclude that for EMG signal of healthy, it has the lowest complexity value and its fluctuation value is in the middle. As for patient with myopathy, both its complexity and fluctuation value are higher than that of healthy. Unlikely, with the highest complexity value, the EMG of patient with neuropathy experiences the lowest fluctuation value. In this paper, complexity of EMG is determined by singular-value decomposition and mutual information method, and a novel effective approach (fluctuation) based on HHT is also proposed. The experiment results show the effectiveness of the proposed approaches and the feasibility of such system. The assessment methods presented here is just a preliminary step that will facilitate the application of EMG in medical purpose, while future extensions will include more sophisticated methods integrated with various evaluation tools.

\section{Acknowledgement}

This research is funded by the Fundamental Research Funds for the Central Universities (Grant No. 2012-IV-088, 2012-ZY-065).

\section{References}

[1] L. Zollo, L. Rossini, M. Bravi, Quantitative evaluation of upper-limb motor control in robot-aided rehabilitation. Med. Biol. Eng. Comput., 2011, 49: 1131-1144.

[2] W. Shi, X. Li, W. Wang, et al., Clinical Effects of Comprehensive Rehabilitation Treatment on Gastronimius Surface Electromyography in Stroke Patients. Chin. J. Rehabil. Med., 2010, 2(25): 103-105.

[3] Y. Y. Huang, K. H. Low, Comprehensive Planning of Robotic Therapy and Assessment of Task-Oriented Functions via Improved QFD Applicable to Hand Rehabilitation. $6^{\text {th }}$ annual IEEE Conference on Automation Science and Engineering. (2010): 252-257.

[4] I. Cikajlo, Z. Matjacic. Directionally Specific Objective Postural Response Assessment Tool for Treatment Evaluation in Stroke Patients. IEEE T. Neur. Sys. Reh., 2009, 1(17): 91-100.

[5] M. Casadio, P. Morasso, A. N. Ide. Measuring functional recovery of hemiparetic subjects during gentle robot therapy. Measurement, (2009):1176-1187.

[6] S. Hussain, S. Q. Xie, G.Y. Liu, Robot assisted treadmill training: Mechanisms and training strategies. Med. Eng. Phys., (2011):527-533.

[7] X.L. Hu, K.Y. Tong, R. Li, J.J. Xue, S.K. Ho, P. Chen, The effects of electromechanical wrist robot assistive system with neuromuscular electrical stimulation for stroke rehabilitation. J. Electromyogr. Kines., (2012). doi:10.1016/j.jelekin.2011.12.010.

[8] M.A. Oskoei, H.S. Hu., Myoelectric control systems - A survey. Biomed. Signal Proces., 2 (2007): 275-294.

[9] T. Yan, W.Y.C. Hui-Chan. Maximum isometric voluntary contraction of ankle dorsiflexors and plantarflexors : comparison of surface electromyography between patients at the acute stage after stroke and age-matched healthy elderly. Chin. J. Phys. Med. Rehabil., (2003) 4(25):212-215.

[10]Q. Cai, D. Sun, Y. tan, et al., SEMG signal change before and after the rehabilitation of patients of hemiplegia. Chin. J. Rehabil. Med., (2008) 4(23): 347-348.

[11] K.M. haithanya, B. Jorge, K. T. Christine. Automatic analysis of EMG during clonus. J. Neurosci. Meth., (2012): 35-43.

[12]N. E. Huang, Introduction to the Hilbert Huang transform and its related mathematical problems, (2005) 1-20. 